Revue des patrimoines

\title{
Étude des peintures murales romanes dans les anciens territoires de Bourgogne : de Berzé-la- Ville à Rome et d'Auxerre à Compostelle
}

Juliette Rollier-Hanselmann

\section{(2) OpenEdition}

\section{Journals}

Édition électronique

URL : http://journals.openedition.org/insitu/10671

DOI : 10.4000/insitu. 10671

ISSN : 1630-7305

Éditeur

Ministère de la culture

Référence électronique

Juliette Rollier-Hanselmann, «Étude des peintures murales romanes dans les anciens territoires de Bourgogne : de Berzé-la-Ville à Rome et d'Auxerre à Compostelle », In Situ [En ligne], 22 | 2013, mis en ligne le 14 novembre 2013, consulté le 19 avril 2019. URL : http://journals.openedition.org/ insitu/10671 ; DOI : 10.4000/insitu.10671

Ce document a été généré automatiquement le 19 avril 2019.

\section{(c) $(1) \Theta \theta$}

In Situ Revues des patrimoines est mis à disposition selon les termes de la licence Creative Commons Attribution - Pas d'Utilisation Commerciale - Pas de Modification 4.0 International. 


\title{
Étude des peintures murales romanes dans les anciens territoires de Bourgogne : de Berzé-la-Ville à Rome et d'Auxerre à Compostelle
}

\author{
Juliette Rollier-Hanselmann
}

L'établissement d'un corpus des peintures murales romanes correspondant aux anciens territoires de Bourgogne permet d'étudier les peintures selon le contexte historique de l'époque et d'établir des comparaisons inédites entre des sites éloignés géographiquement. Les échanges entre ateliers, le long de voies de pèlerinage, ont favorisé les liens avec certains sites de l'ouest de la France, tandis que les relations historiques étroites entre Cluny, la Catalogne et la Castille-Leon expliquent qu'un tiers des peintures soit fortement influencé par l'art de la péninsule ibérique.

\section{La peinture clunisienne à son apogée : Berzé-la-Ville, La Chapelle-des-Moines}

Il ne subsiste que très peu d'éléments permettant de connaître la peinture clunisienne. La Chapelle-des-Moines et le Lectionnaire de Cluny ${ }^{1}$ constituent des jalons importants de la production artistique du début du XII siècle. Si la documentation indique que l'abbé Hugues de Semur (1049-1109) s'est fait construire cette petite chapelle privée, la datation des peintures reste incertaine entre 1109 et 1120 . Cet exemple est unique dans la région, tant par les sujets iconographiques que par le style fortement inspiré des milieux romains ${ }^{2}$. La composition est une savante construction intellectuelle qui reflète la pensée clunisienne ${ }^{3}$. Pour l'abside, l'artiste et le commanditaire ont choisi la traditio legis et clavium, thème rare dans la peinture murale romane et unique dans notre corpus. Dans la chapelle privée de l'abbé de Cluny, il s'agissait de montrer la primauté du siège apostolique et l'importance de Pierre et Paul illustrant implicitement le privilège 
d'exemption monastique. Les papes Grégoire VII et Urbain II ont utilisé Cluny comme un symbole de propagande de la liberté de l'Église.

Figure 1

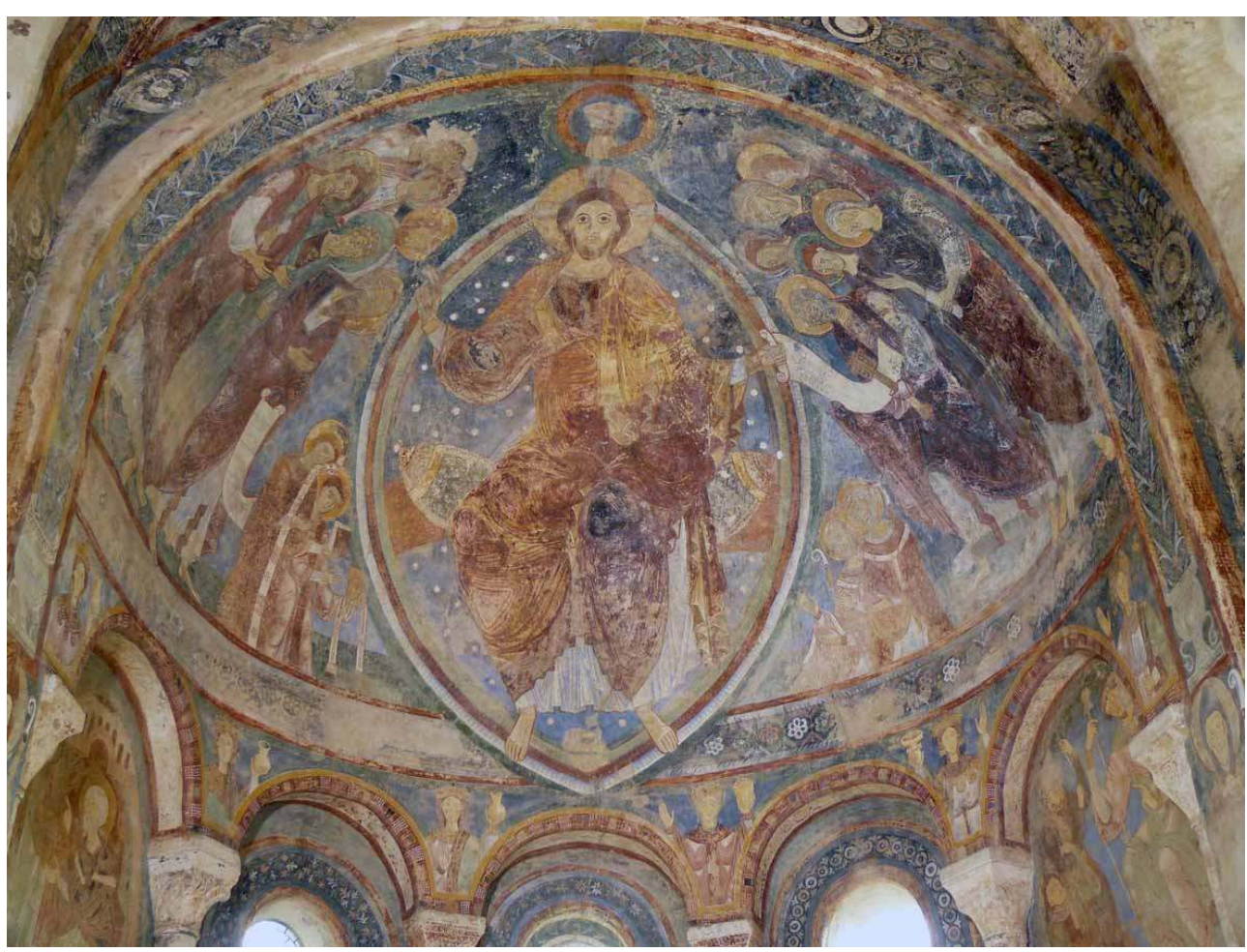

Berzé-la-Ville, la Chapelle-des-Moines, vue de l'abside.

PHOT. ROLLIER, J. ( ) J. ROLLIER.

3 La traditio legis et clavium - Remise de la loi et des clefs à saint Pierre et saint Paul - de Berzé-laVille (fig. $\mathbf{n}^{\circ} \mathbf{1}$ ) est une synthèse de modèles anciens ${ }^{4}$ qui s'inscrit dans un vaste mouvement de renouveau, actif à la fin du XI et au début du XII siècle à Rome et dans de nombreux lieux. Les exemples ne manquaient pas pour inspirer le peintre et le commanditaire, comme le mausolée de Sainte-Constance à Rome (mosaïque datée vers 350), les sarcophages de Rome, Milan ou Ravenne. Il ne s'agit pas d'une reprise directe des exemples italiens, ni de ceux du sud de la France, mais plutôt d'une réinterprétation de ceux-ci. Le sarcophage dit du Christ docteur remettant la loi à saint Pierre (Musée de l'Arles antique) (fig. $\mathbf{n}^{\circ} \mathbf{2}$ ) provenant de l'église Saint-Honorat des Alyscamps, rattachée au site clunisien de Saint-Victor de Marseille (1040-1044) ${ }^{5}$, s'inscrit dans les modèles possibles puisqu'il a été remployé au revers de l'autel majeur de l'église Saint-Honorat ${ }^{6}$. 


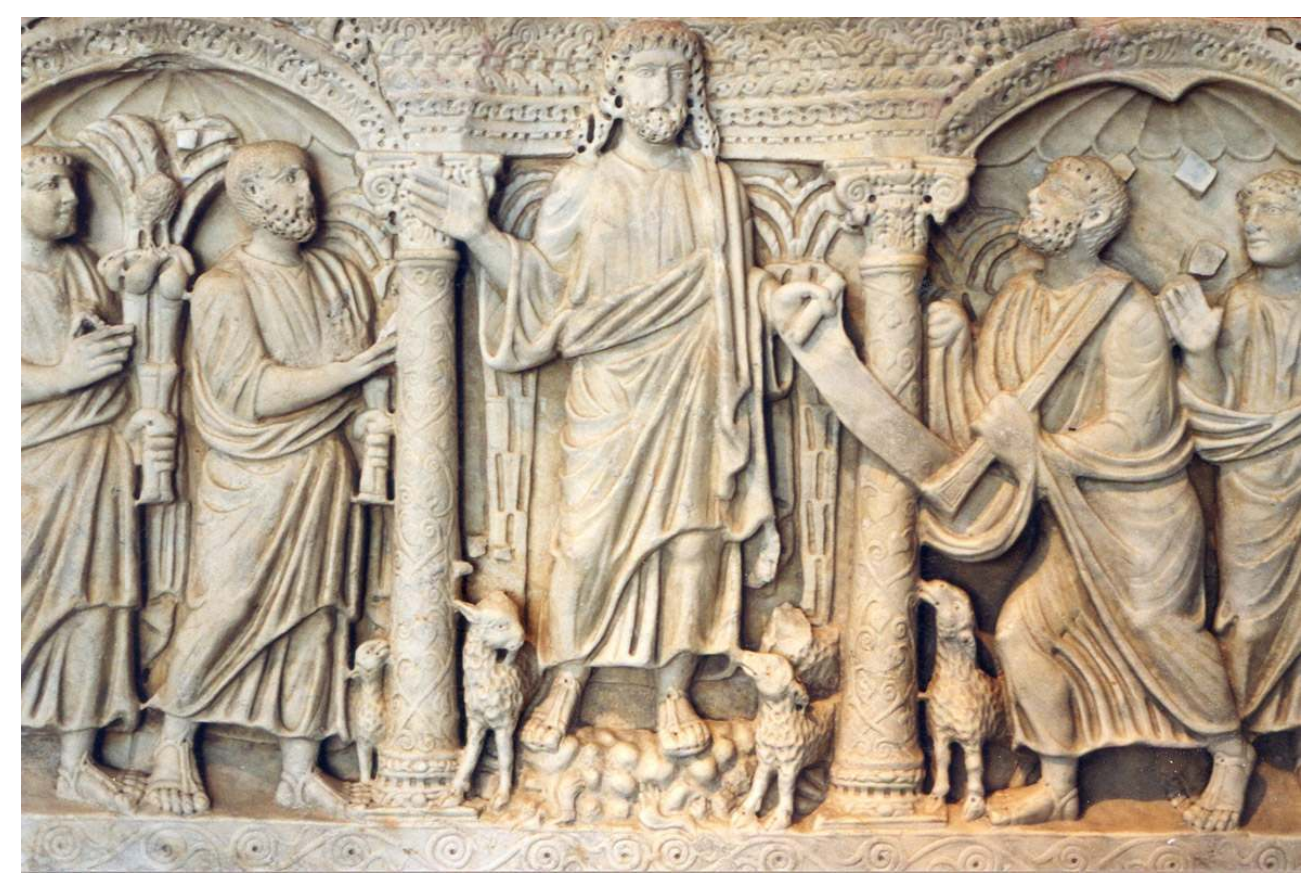

Musée de l'Arles antique, Sarcophage du Christ docteur.

PHOT. ROLLIER, J. ( ) J. ROLLIER.

La valeur politique de l'image est perceptible sur le baldaquin de Saint-Ambroise de Milan que les Clunisiens ont dû connaître. Ce ciborium en stuc polychrome, construit sous l'évêque Angilbert II, vers le milieu du IX ${ }^{\mathrm{e}}$ siècle, présente une traditio legis orientée vers la nef et donc destinée aux fidèles. Le thème s'adresse à une large foule et rappelle le rôle de l'évêque Angilbert ll, bien connu pour son adhésion aux programmes de réforme ${ }^{7}$. Dans le même ordre d'idée, à l'ouest de Cluny, dans la ville de Nevers, un petit portail ${ }^{8}$, autrefois situé à l'entrée de l'église clunisienne de Saint-Sauveur, présentait l'épisode de la Remise des clés à saint Pierre (traditio clavium) (fig. $\mathbf{n}^{\circ} \mathbf{3}$ ). Le Christ est assis de profil, plaçant Pierre en position centrale, pour illustrer clairement le lien avec la papauté. Le portail se situait à proximité de la rivière, à l'endroit où les nombreux pèlerins de la voie de Limoges traversaient la Loire. 


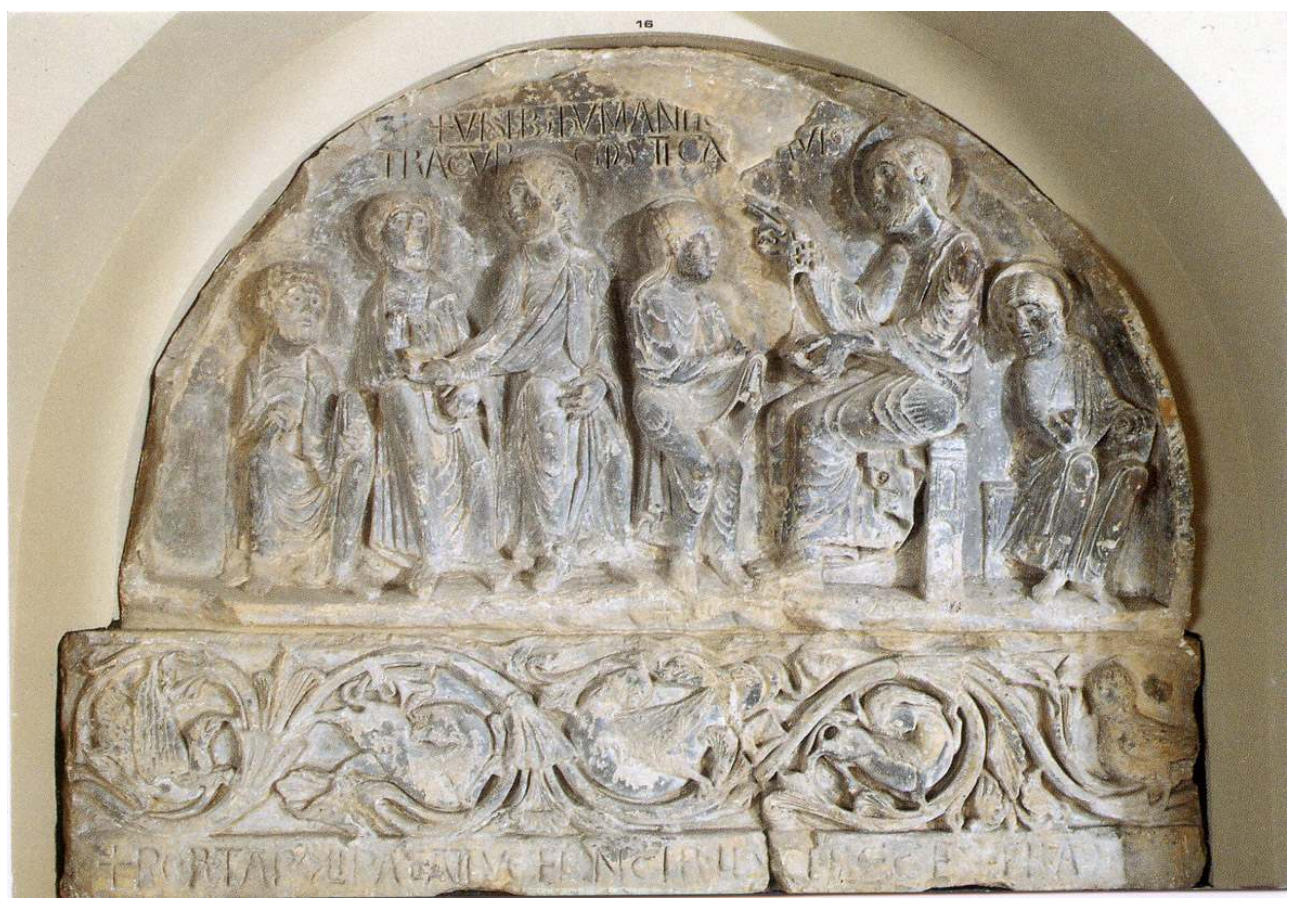

Nevers, église Saint-Sauveur, musée de la Porte du Croux. Carte postale.

Phot. Rollier, J. (c) Nevers, musée de la Porte du Croux.

5 L'utilisation du thème est tout autre dans l'ancien royaume de Bourgogne, près de Lyon, à Saint-Chef-en-Dauphiné (Isère) où la traditio legis à trois personnages occupe les ébrasements d'une baie du bras nord du transept, en relation avec l'usage baptismal inhabituel de cette partie de l'édifice ${ }^{9}$. Cette typologie est issue de modèles paléochrétiens, tel celui du baptistère de Naples par exemple ${ }^{10}$. À Saint-Chef la Remise de la loi s'insère dans le programme pictural du transept qui comporte une chapelle à deux niveaux ${ }^{11}$. L'élaboration de cet ensemble atypique a demandé un effort particulier au niveau de la logique interne des différents espaces peints qui devaient répondre aux usages monastiques et paroissiaux. Or les peintures se situent soit sous l'épiscopat de Warmond (1076-1082), qui fut un disciple de l'abbé Hugues de Cluny, soit sous l'abbatiat de Guy de Bourgogne (1088-1119), qui devint pape en 1119 sous le nom de Callixte II. De telles figures ont favorisé une réflexion approfondie et renouvelée autour de la création de ce cycle peint.

6 L'abside de Berzé-la-Ville ne trouve pas d'équivalent dans d'autres régions de France. Le seul autre exemple peint de traditio legis avec le cortège apostolique complet subsiste dans la petite église du prieuré bénédictin de Saint-Gilles de Montoire ${ }^{12}$, dans le bras sud du transept, dont la peinture est datée vers le milieu du XII ${ }^{\mathrm{e}}$ siècle. D'ailleurs la présence des saints Laurent et Blaise pourrait indiquer que l'artiste a réadapté des thèmes présentés dans la Chapelle-des-Moines. Les liens entre Montoire et Cluny doivent cependant être éclaircis, afin de mieux comprendre de tels transferts. 


\section{Étude technique des peintures, étendue des repeints}

7 L'abside de la Chapelle-des-Moines a été entièrement repeinte, à l'époque gothique probablement, en correspondance avec les peintures de la nef (fig. $\left.\mathbf{n}^{\circ} \mathbf{4}\right)$. La superposition stratigraphique est clairement visible au sommet de l'abside où des fleurettes rouges recouvrent le décor roman. Lors de la découverte des peintures en 1887, une méconnaissance des strates a conduit au dégagement imparfait des deux couches, montrant tantôt la couche ancienne, tantôt de larges zones repeintes. L'étude technique et les analyses de laboratoire ont apporté toute la clarté sur ce point. À l'origine le Christ roman portait un manteau jaune lumineux, qui a ensuite été repeint en rouge ${ }^{13}$. Le Christ vêtu de jaune renvoyait aux modèles paléochrétiens et carolingiens (Saint-Cosme-etSaint-Damien, Sainte-Cécile in Trastevere, Bible de Vivien, Sacramentaire de Metz) ${ }^{14}$. L'or est le reflet de la lumière divine ${ }^{15}$, selon Bède le Vénérable (De templo Salomonis 12), Bruno de Segni (Sent.4, De templo Salomonis) et Honorius d'Autun (Expos. In Cant. 5, De Imagine Mundi) ${ }^{16}$. En peinture murale, le jaune sert de substitut à l'or et sa luminosité est mise en valeur par l'utilisation abondante du bleu de lapis-lazuli pour les fonds. Liz James ${ }^{17} \mathrm{a}$ montré l'importance du choix des couleurs, ainsi que leur manipulation selon la lumière dans les peintures byzantines, pratiques qui ont probablement été mises en œuvre dans la Chapelle-des-Moines.

Figure 4

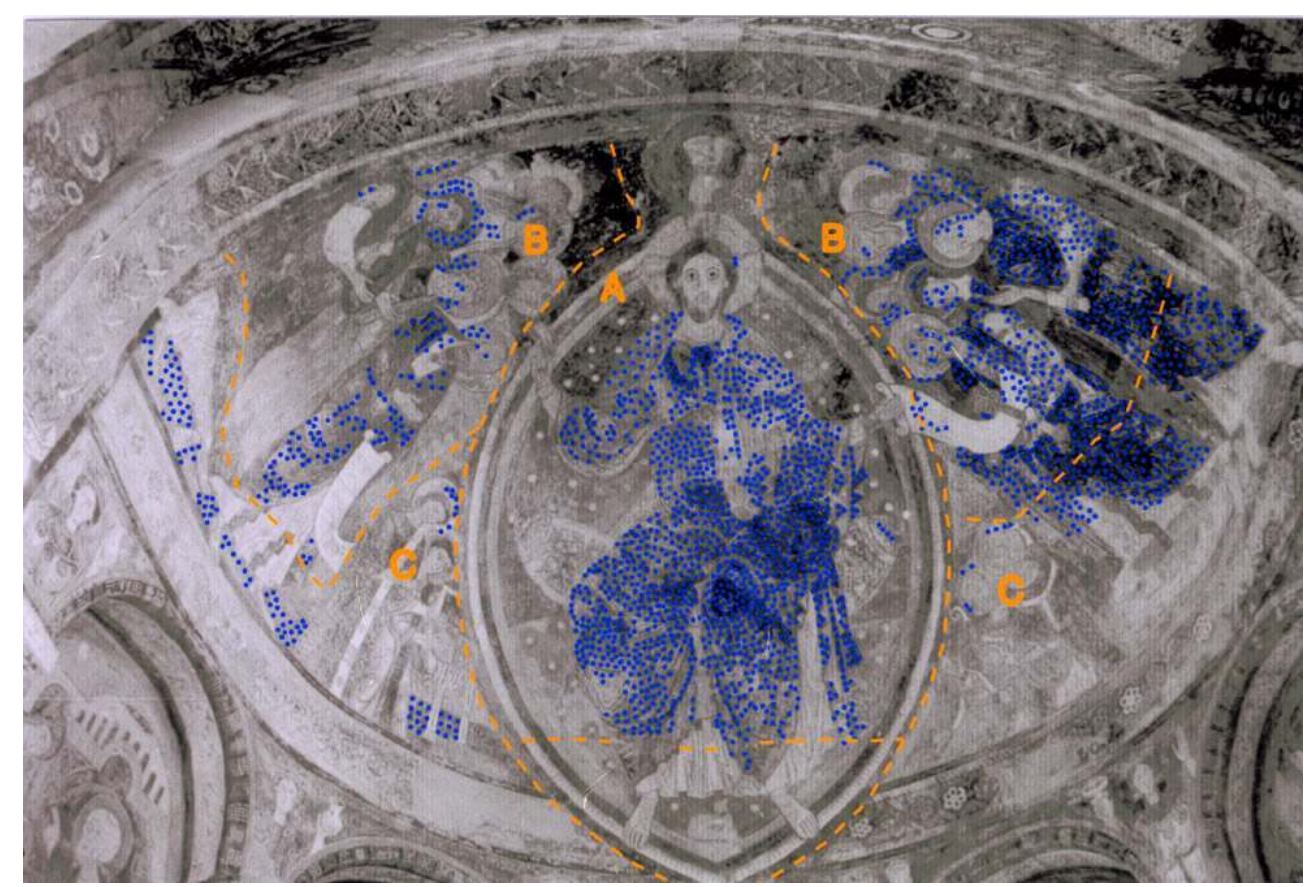

Berzé-la-Ville, la Chapelle-des-Moines, schéma des repeints.

Phot. Rollier, J. (c) J. Rollier.

8 D'autres exemples de Christ vêtu d'un manteau jaune lumineux sont connus pour l'époque romane, notamment sur la voûte de Saint-Savin-sur-Gartempe (cycle de la Genèse), ainsi que dans la crypte ${ }^{18}$. En Italie centrale, les images de Christ vêtu de jaune imitant l'or sont fréquentes, notamment dans le Latium, à Castel Sant' Elia (basilique Saint-Anastase) et Tivoli (San Silvestro). D'autres œuvres se situent à Rome, dans le milieu 
réformé du monastère féminin de San Gregorio Nazanzieno (panneau du Jugement dernier ${ }^{19}$, daté entre 1061 et 1071), dans les mosaïques de l'église de San Clemente ${ }^{20}$ (vers 1118).

9 L'étude stratigraphique et technique des peintures a apporté bien d'autres informations, concernant la composition des enduits, la palette picturale, l'utilisation de pigments qui ont noirci avec le temps. Les analyses réalisées par le Laboratoire de recherche des Monuments historiques (Paulette Hugon) et les identifications de pigments faites au Raman par Claude Coupry au LADIR (Laboratoire de dynamique, Interactions et Réactivité, Thiais) ont fourni de nombreuses données sur les matériaux d'origine, leurs altérations et les repeints ${ }^{21}$.

\section{Modélisation 3D et étude des modules de construction}

10 Le peintre de Berzé-la-Ville a réalisé une composition extrêmement dense, avec plus de quarante personnages, dans un espace restreint. Une organisation interne stricte et une préparation précise des peintures ont certainement précédé le chantier. La mise en place de la mandorle devait être faite à l'aide d'une grande corde sur une surface courbe et les espaces entre les différents groupes de personnages calculés à l'avance. Le Christ central se détache nettement des apôtres, resserrés de part et d'autre, tout en laissant un espace distinct aux deux groupes de personnages placés aux pieds du Christ (d'une part deux abbés, d'autre part les saints Laurent et Vincent). La lecture de l'ensemble reste ainsi extrêmement claire. De tels principes de composition et une telle densité thématique peuvent également être observés sur certains portails, notamment à Vézelay, au tympan central (daté vers 1130-40) où la position du Christ est pratiquement identique à celle de Berzé-la-Ville.

Une logique géométrique interne a permis de placer la quarantaine de personnages dans un espace réduit (dimensions de l'abside : L. 2,94 m x 1. 3,26 m x H 6,62 m). La photomodélisation en 3D permet de réfléchir concrètement aux méthodes d'élaboration des peintures. La construction intérieure de la chapelle semble avoir été élaborée à partir de deux sphères superposées, dont chacune contient un carré. La moitié de ce carré représente la hauteur du soubassement, ce qui sous-entend vraisemblablement la mise en place d'un module de base. Une première solution (fig. $\mathbf{n}^{\circ} 5$ ) consisterait à placer le cercle supérieur en correspondance avec la bordure végétale qui entoure l'arc absidal, ce qui expliquerait les corrections de ces bordures au niveau des murs latéraux ${ }^{22}$. En effet, la bordure décorative ne trouve pas suffisamment de place à la retombée de l'arc, si bien que le décor déborde de l'abside. Dans ce cas nous constatons que l'artiste a rencontré des difficultés dans la mise en place de son schéma de construction. 
Figure 5
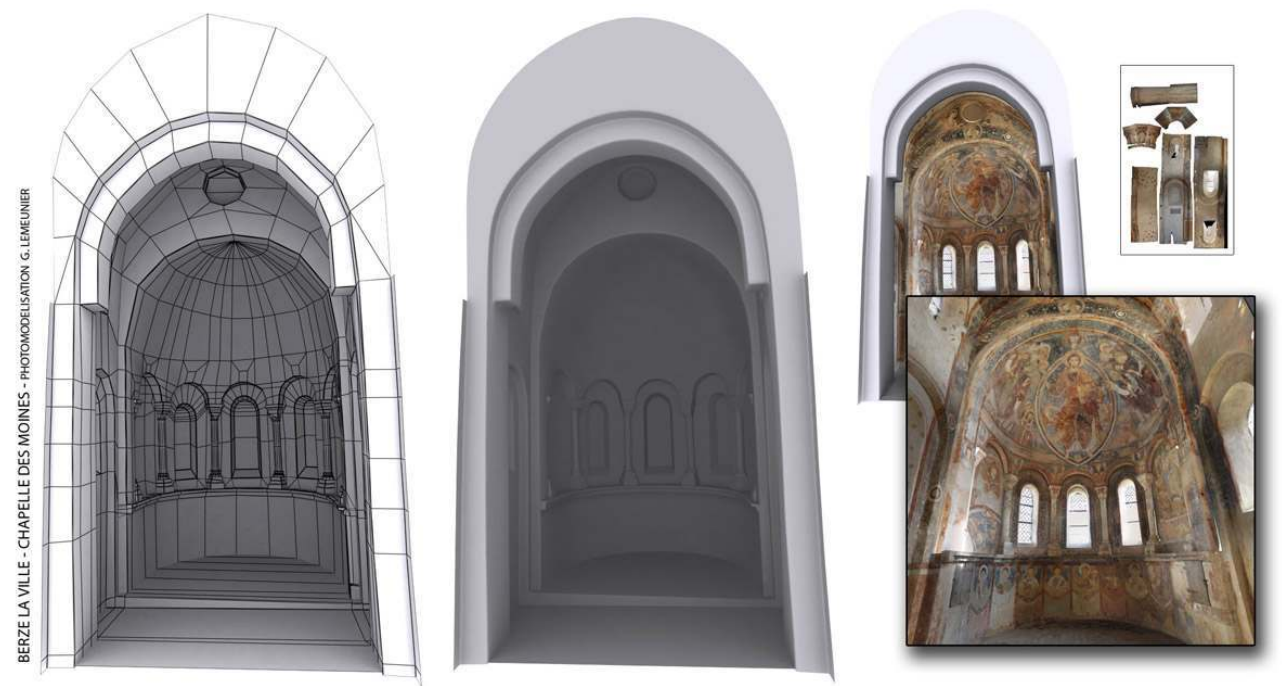

Berzé-la-Ville, la Chapelle-des-Moines, photomodélisation 3D.

Phot. Lemeunier, G. @ G G. Lemeunier.

Une autre solution tient compte d'une éventuelle réfection du dallage, de telle manière que les deux sphères soient placées sous l'abside, avec deux carrés superposés en relation avec les chapiteaux qui délimitent la jonction entre les murs et la conque, méthode qui paraît plus imprécise pour la mise en place des peintures.

Nous constatons également que les huit saints du soubassement ont été placés dans des rectangles verticaux qui respectent les proportions du nombre d'or.

Ces quelques observations mériteraient d'être approfondies et une véritable étude serait nécessaire pour mieux comprendre le système de construction picturale. Il manque également une étude archéologique des élévations pour connaître les éventuelles réfections de la chapelle.

L'étude des visages permet aussi de faire un certain nombre de constatations. Pour celui du Christ (fig. $\mathbf{n}^{\circ} \mathbf{6}$ ), qui se trouve sur une surface pratiquement plane, il semblerait qu'un système à trois cercles ait été utilisé, la longueur du nez correspondant à l'intervalle entre les deux pupilles et au rayon du premier cercle. Le même principe a été appliqué aux têtes des apôtres, ce qui permettait d'obtenir une grande homogénéité de composition. L'utilisation d'un système à trois cercles apparaît encore plus clairement sur la tête féminine dont la couche picturale usée laisse transparaître les tracés préparatoires (scène du martyre de saint Blaise). Le cercle intermédiaire, autrefois caché sous le voile, est visible dans les lacunes de la couche picturale. 


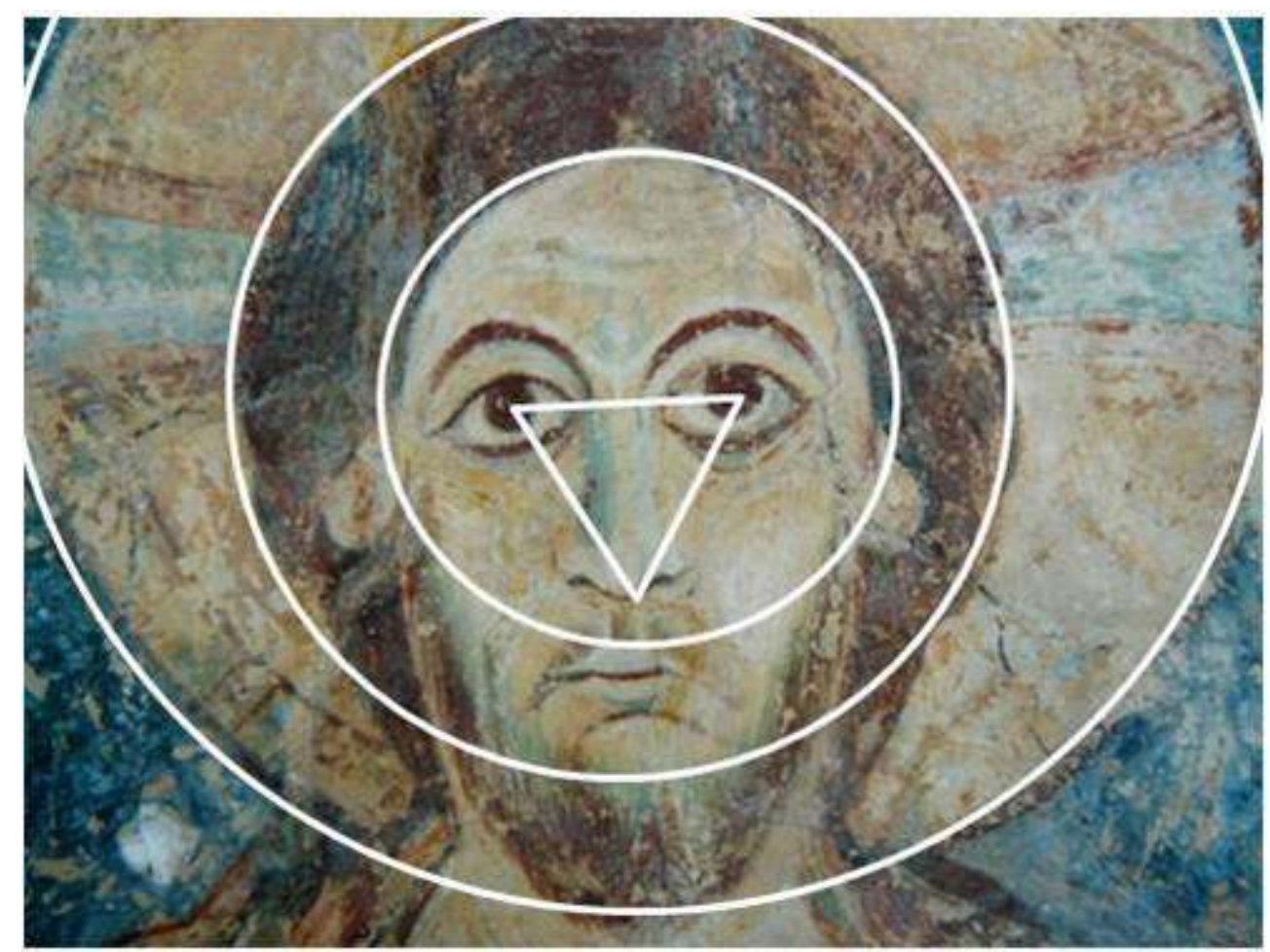

Berzé-la-Ville, la Chapelle-des-Moines, visage du Christ.

Phot. Rollier, J. (c) J. Rollier.

D’autres exemples de modules géométriques ont été observés ailleurs en France, notamment dans le cloître de Saint-Aubin à Angers (XII ${ }^{\mathrm{e}}$ siècle) pour l'image de la Jérusalem céleste. Le schéma régulateur proposé par Christian Davy ${ }^{23}$ montre que le cercle et le carré ont servi de modules de base à cette composition hautement symbolique. Ici le nombre d'or est mis en œuvre à deux reprises. Un autre cas intéressant est celui de l'église Saint-Hilaire d'Oisé (Sarthe) où un schéma régulateur semble avoir été utilisé pour le Baptême du Christ. Si la symétrie est une méthode simple pour disposer les personnages dans une composition, l'utilisation de deux triangles équilatéraux dans un cercle permet de subdiviser l'espace en douze compartiments et de placer les personnages selon une organisation précise. Le centre du cercle coïncide avec la base de la mandorle de l'âme de saint Hilaire, qui possède un rayon égal à la distance de la tour symbolisant l'entrée de la Jérusalem céleste.

17 L'utilisation de la géométrie dans l'élaboration de peintures murales romanes a également été constatée dans d'autres sites d'Europe ${ }^{24}$. Les recherches dans ce domaine restent cependant rares. L'intérêt des études interdisciplinaires réside dans le fait que l'imagerie 3D offre de nouveaux outils de recherche pour l'histoire de l'art et l'étude des techniques picturales médiévales.

\section{Étude du corpus bourguignon}

Le corpus des anciens territoires de Bourgogne (ancien duché, ancien royaume de Bourgogne) s'étend d'Auxerre à Bâle pour la limite nord, et de Nevers à la Provence pour 
la partie méridionale. Le corpus subsistant étant très hétéroclite au point de vue stylistique, il est difficile de dégager les caractéristiques de son évolution. Les comparaisons entre sites restent limitées. Certaines œuvres semblent attester de relations avec des ateliers de l'ouest, comme celles d'Autun, Auxerre et Anzy-le-Duc. D'autres peintures sont nettement plus difficiles à classer en raison d'influences mixtes, comme Tournus, Nevers et les Allinges. D'une manière générale, il s'agit de lieux non clunisiens, répartis entre le diocèse d'Autun, qui s'étendait alors jusqu'en Côte d'or, et les diocèses d'Auxerre, Chalon et Mâcon. La Savoie et la Haute-Savoie sont également concernées.

19 La plupart des peintures se trouvent dans des sites placés le long des voies de pèlerinage ce qui devait faciliter les échanges entre les différents lieux. Les similitudes stylistiques qui apparaissent entre certains sites sont étonnantes et permettent probablement d'identifier pour la première fois des ateliers itinérants.

Un tiers des sites étudiés présente des caractéristiques stylistiques qui attestent des liens avec l'ouest de la France. Dans certains cas les comparaisons s'établissent ponctuellement à partir de quelques détails, entre Autun (Saint-Nicolas-lès-Marchaux) et Saint-Gilles-deMontoire (fig. $\left.\mathbf{n}^{\circ} \mathbf{7}\right)$ (fig. $\mathbf{n}^{\circ} \mathbf{8}$ ), entre Tournus (avant-nef) et Saint-Savin, entre Anzy-le-Duc et Brinay-sur-Cher.

\section{Figure 7}

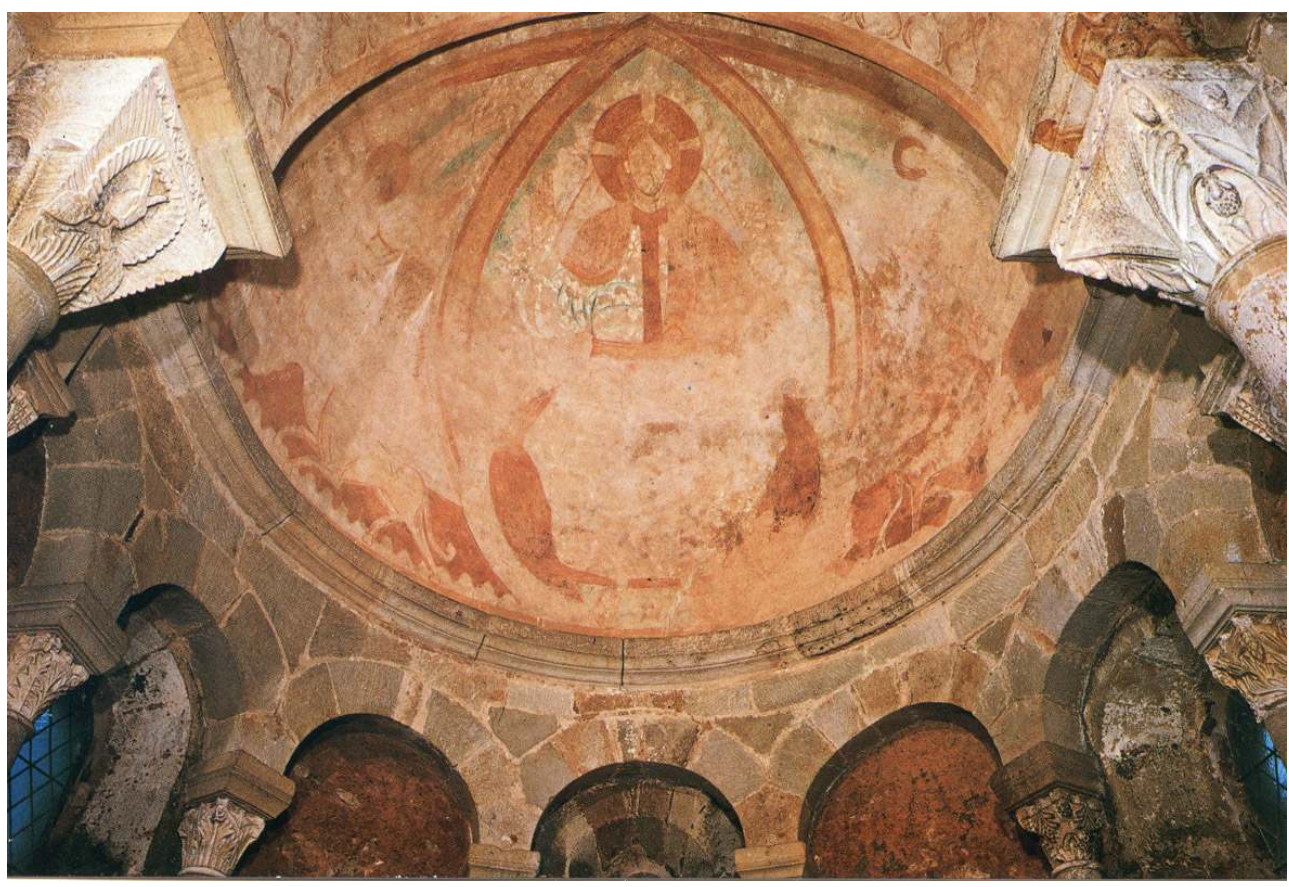

Autun, église Saint-Nicolas-lès-Marchaux.

PHOT. ROLLIER, J. @ J. ROLLIER. 


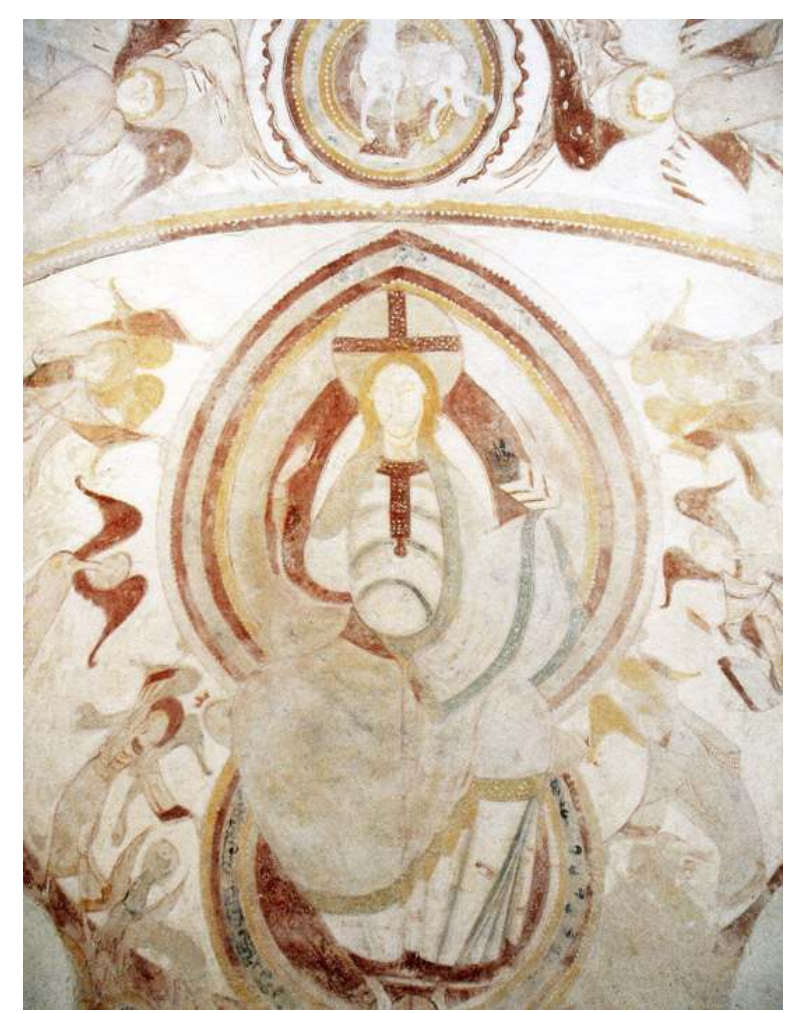

Montoire, église Saint-Gilles, abside centrale.

Repro. Rollier, J. ( C C. Davy.

L'exemple de la crypte de la cathédrale Saint-Étienne d'Auxerre est très intéressant car ses peintures présentent des ressemblances avec celles des églises Saint-Hippolyte de Combertault (Côte d'Or) et Saint-Laurent de Neuvy-Pailloux (Indre). L'identification d'un atelier itinérant paraît ici possible, d'autant plus que les deux sites d'Auxerre et NeuvyPailloux sont relativement proches le long de la grande voie de pèlerinage de Limoges (via Lemovicensis), reliant Auxerre à Châteauroux, en passant par Vézelay, la Charité-sur-Loire, Bourges. Le petit village de Combertault est également sur un important axe de circulation entre Dijon et Mâcon.

Si certaines relations indiquent une influence de l'ouest de la France, il existe aussi une influence anglaise, notamment par les liens entre les peintures de Combertault et la Bible de Cîteaux ou d'Étienne Harding 25 , datée vers 1109, issue du scriptorium cistercien. La liberté figurative très nouvelle du cycle de saint Hippolyte et la mode vestimentaire des personnages laïcs incitent à une datation vers le début du XII ${ }^{\mathrm{e}}$ siècle.

Les questions de datation soulèvent de nombreuses questions. Dans l'état actuel des connaissances, une classification très générale peut être faite entre les sites du début et de la fin du XII ${ }^{e}$ siècle. Des recherches historiques sont nécessaires pour chacun site, afin de déterminer les faits qui ont pu influer sur la création artistique. À titre d'exemple, l'absence d'étude archéologique pour dater l'église Saint-Nicolas-lès-Marchaux à Autun, rend la datation des peintures incertaine. Il existe cependant une similitude étroite entre le Christ autunois et celui de l'abside centrale de Saint-Gilles de Montoire, daté de la fin XI ème-début XII ${ }^{\mathrm{e}}$ siècle, ce qui situe provisoirement l'abside d'Autun à la même période. 
Dans les cas mieux étudiés, tel le Christ-cavalier de la cathédrale d'Auxerre, les spécialistes avancent une datation qui varie entre 1030 et $1052-1076^{26}$, ou plus tardivement sous l'évêque Humbaud qui fut un mécène actif entre 1092-1114 ${ }^{27}$. Cette dernière fourchette, plus plausible, permet peut-être d'établir une relation entre l'image des cavaliers et l'appel à la première croisade en 1095.

De la seconde moitié du XII ${ }^{e}$ siècle, sont datables les ensembles importants des absides de Sussey-le-Maupas (Côte d'Or), les Allinges (Haute-Savoie), Nevers (Nièvre), Saulzet (Drôme), l'île Barbe à Lyon, ainsi que l'avant-nef de Payerne (Suisse romande) et les peintures détruites de l'ancien réfectoire de Charlieu. Si la restauration très marquée des absides d'Anzy-le-Duc et de Nevers ${ }^{28}$ rend l'analyse incertaine, il apparaît que ces décors, ainsi que ceux de Charlieu, Saulzet et Payerne appartiennent à une époque de transition entre art roman et art gothique. À la fin de notre série se situent l'église de Moutiers (Yonne), dont les décors sont volontairement archaïsants, et Saint-Martin d'Aime (Savoie) qui rappelle des productions italiennes des années 1200.

Nous aimerions également pouvoir situer de manière plus claire la production des anciens territoires bourguignons dans le paysage artistique français, mais les difficultés sont multiples. Certaines peintures romanes de France n'ont pas encore fait l'objet d'inventaires ${ }^{29}$ systématiques et les études plus poussées restent encore le privilège de quelques régions, comme le Poitou ${ }^{30}$, l'Auvergne ${ }^{31}$, les Pays de Loire $^{32}$, le Centre ${ }^{33}$ ou les Pyrénées $^{34}$. Des inventaires et des thèses en cours apporteront des compléments à notre thématique.

\section{De Cluny à la péninsule ibérique}

Un tiers des peintures du corpus des anciens territoires de Bourgogne présentent des caractéristiques stylistiques hispanisantes, que l'on peut expliquer par les faits historiques. Les relations entre Cluny et la péninsule ibérique sont déjà bien installées sous l'abbé Odilon (994-1049) qui participe activement à la lutte contre les Sarrasins. Au début $\mathrm{du} \mathrm{XI}{ }^{\mathrm{e}}$ siècle beaucoup de pèlerins partent vers Compostelle et les grands lignages du mâconnais sont constamment présents en terre ibérique ${ }^{35}$.

alliances sont conclues entre les grandes lignées françaises et les souverains de León, notamment par les «mariages bourguignons». Ainsi Alphonse VI épousa d'abord Agnès (1074-1078), fille du duc Guillaume VI d'Aquitaine, qui mourut rapidement. Sa deuxième épouse fut Constance, fille du premier duc capétien de Bourgogne, Robert, mais également la nièce de l'abbé Hugues.

Le roi Alphonse VI chercha à marquer la route du pèlerinage par des constructions ${ }^{36}$. L'introduction du rite romain, exigé par le pape Grégoire VII, et le développement du pèlerinage vers Saint-Jacques, ont fait entrer la péninsule dans l'orbite européenne, ce qui a permis de rétablir la situation face à l'Islam ${ }^{37}$.

Les relations entre Cluny et la Catalogne sont anciennes. Certaines abbayes de Catalogne étaient en lien direct avec Rome, certaines étant même des relais de Saint-Pierre de Rome.

L'identification par Meyer Schapiro du manuscrit clunisien de Saint-Ildefonse (Bibl. palatine de Parme $)^{38}$ et l'importance des apports ibériques dans le style du peintre ouvrent de nouvelles voies de recherche. Ce manuscrit a probablement été réalisé pour un commanditaire important, comme le roi Alphonse VI de Castille et León (1072-1109), 
ce qui permet d'en situer la date. Lorenza Cocchetti Pratesi ${ }^{39}$ a montré l'importance des apports ibériques dans le style du peintre et les rapprochements qu'elle établit avec les peintures murales de Saint-Clément de Tahull et Sainte-Eugénie d'Argolell lui permettent de situer la réalisation du manuscrit au monastère de Saint-Benoît de Ripoll, alors fortement imprégné d'art ottonien.

Dans ces conditions, il n'est pas étonnant de constater qu'au nord de Cluny, dans la petite église Saint-Martin d'Ougy ${ }^{40}$, un portrait de saint Philippe rappelle l'art de Saint-Clément de Tahull, dont les peintures sont datées vers 1123. De telles similitudes s'inscrivent dans une période d'échanges étroits entre les territoires de Bourgogne et les Pyrénées. L'église de Saint-Clément de Tahull se situe à l'époque de Raymond (1104-1126), qui fut également prieur de Saint-Sernin de Toulouse (1100). Les peintures murales sont dues à un artiste qui intègre des influences diverses, venant de Lombardie et de la sculpture toulousaine ${ }^{41}$.

\section{Le Christ à la faucille de Saint-Nizier de Burnand (Saône-et-Loire)}

L'église de Burnand, située à quelques kilomètres au nord de Cluny, de petites dimensions, comporte une nef unique et une travée précédant l'abside semi-circulaire. L'édifice date du milieu ou de la seconde moitié du $\mathrm{XI}^{\mathrm{e}}$ siècle ${ }^{42}$.

Les peintures de l'abside étaient cachées sous plusieurs couches de badigeon et ont été restaurées de manière exemplaire par Anne Féton ${ }^{43}$. Dans la conque une couche romane est partiellement recouverte d'une peinture $\mathrm{du} \mathrm{XV}^{\mathrm{e}}$ siècle, ce qui a compliqué l'intervention. La majeure partie des peintures est restée en place $^{44}$, tandis que d'autres fragments ont été déposés et sont présentés sur des panneaux séparés (réserves du musée archéologique de Dijon).

Le Christ en majesté d'époque romane est assis dans une double mandorle en forme de huit, dont le cercle inférieur lui sert de siège (fig. $\mathbf{n}^{\circ} \mathbf{9}$ ). Il est partiellement recouvert par le Christ gothique, entouré des attributs de son martyre (croix, instruments de la Passion). Les quatre symboles des évangélistes d'époque romane sont bien préservés. Deux personnages se trouvent aux extrémités de l'abside, éléments qui sont recouverts par les deux évangélistes d'époque gothique (ange de saint Matthieu à gauche et aigle de saint Jean à droite). La restauratrice Anne Féton a effectué un relevé des deux strates peintes de l'abside, ce qui permet de dissocier et de comprendre les couches. 


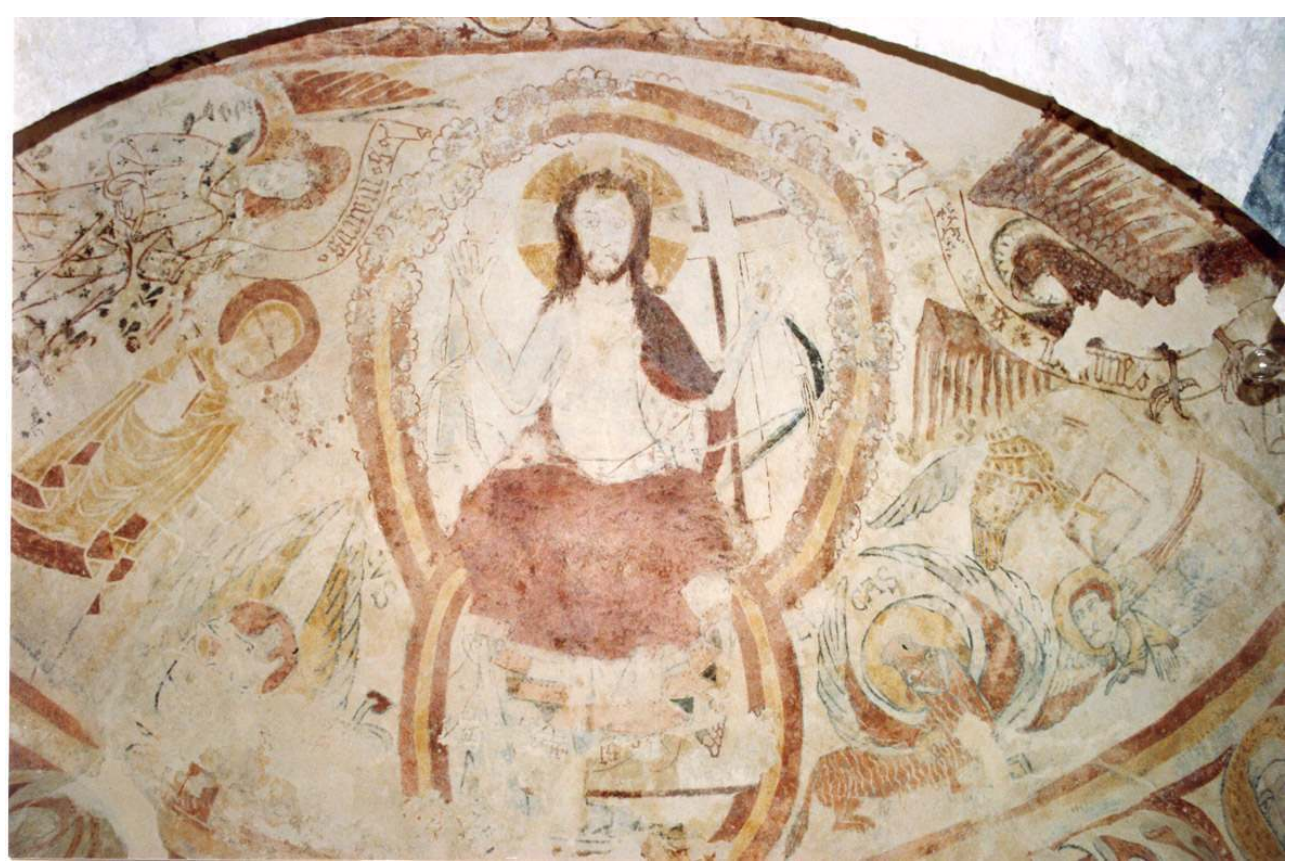

Burnand, église Saint-Nizier, vue de l'abside.

PHOT. ROLLIER, J. @ J. ROLLIER.

Malgré quelques faiblesses dans la mise en place de la composition, avec une mandorle tracée à main levée, l'intérêt de cette abside réside dans l'iconographie très particulière $\mathrm{du}$ Christ roman, qui tient une faucille. Ce type de composition, unique en Bourgogne, semble issu d'un modèle iconographique ibérique, comme le commentaire de l'Apocalypse de Beatus de Liébana, conservé à la bibliothèque de la cathédrale de Burgo de Osma (province de Soria, Castille- León) ${ }^{45}$ qui contient un feuillet (ms 1, fol. 131v) où le Christ trône sur un nuage, tenant une faucille dans sa main gauche. Il est accompagné par des anges occupés aux travaux de vendanges et de moisson. Ce manuscrit, attribué à l'évêque Etherius, est daté de 1086 (fol. 10v) ; il est signé par le scribe Pierre, au fol. 138v (Petrus, clericus scripsit) et le peintre Martin est cité au fol. 163 (Martini peccatoris). Comme l'indique le titulus du feuillet $131 \mathrm{v}$ il s'agit d'une scène de vendange ${ }^{46}$ qui correspond à un extrait du récit de l'Apocalypse (XIV, 14-20).

D'autres détails peints dans l'abside de Burnand ${ }^{47}$ nous ramènent en Catalogne, comme le pelage ondulé du taureau de saint Luc connu dans la Bible de Roda (Bibl. nat., ms. lat. 6), dans le feuillet illustrant la vie de Daniel (vol. III, fol. 66). La datation de cette Bible se situe dans la première moitié du XI $\mathrm{Xiècle}^{48}$.

De même, des détails vestimentaires des apôtres de Burnand (fig. $\mathbf{n}^{\circ} \mathbf{1 0}$ ) se retrouvent sur le devant d'autel de la Seu d'Urgell (début XII ${ }^{\mathrm{e}}$ siècle). Les vêtements sont peints en aplats uniformes, jaune, rouge ou gris, rehaussés de plis épais. Le peintre de Burnand donne peu de mouvement aux tissus et pose des rehauts en série de points, selon la même technique picturale que celle du devant d'autel. Malgré l'usure des peintures murales, nous constatons que le peintre a simplifié certains détails par rapport au panneau sur bois. $\mathrm{Si}$ le maitre d'Urgell a profité de tous les avantages d'un travail en atelier, le muraliste a œuvré dans des conditions plus précaires, sur un chantier perdu en pleine campagne, avec des matériaux moins raffinés. Le peintre de Burnand est plus modeste et manque 
d'organisation dans sa composition, comme l'indiquent les registres irréguliers (montants et descendants) qui n'ont pas été tracés au cordeau.

Figure 10

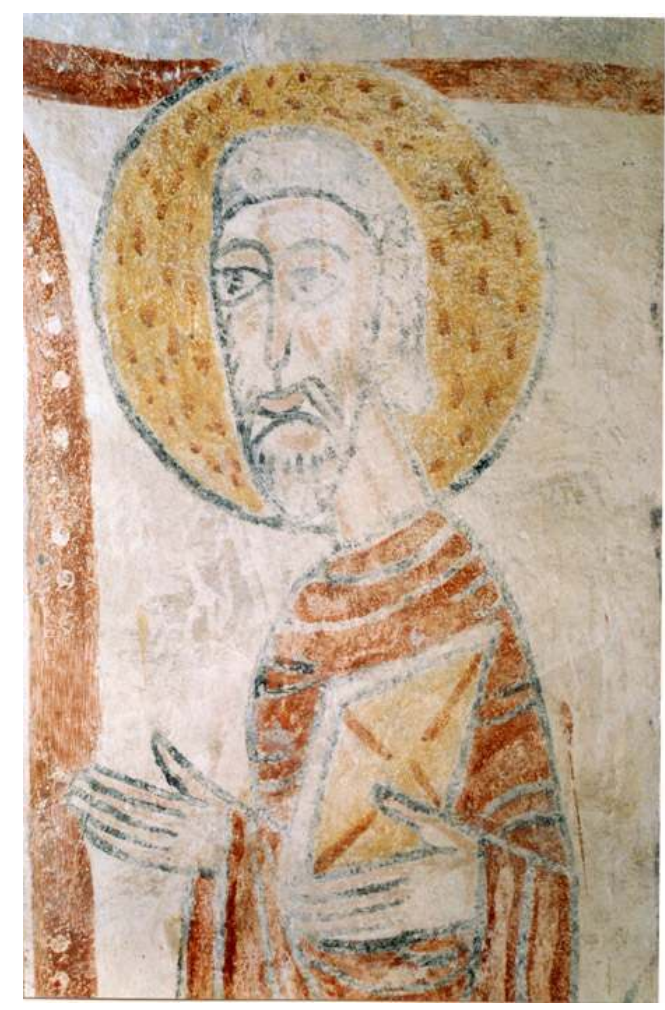

Burnand, église Saint-Nizier, détail d'un apôtre.

PHOT. ROLLIER, J. ( ) J. ROLLIER.

Les visages, aux grands yeux écarquillés, sont très schématiques, avec des fonds roses, rehaussés de lignes roses plus soutenues et de contours noirs simples, sans dégradé. Les détails du visage sont marqués avec un coup de pinceau épais, en rose moyen (pommettes roses, front, menton). La technique picturale des visages et des mains est similaire à celle du devant d'autel catalan, avec des caractéristiques qui perdurent à Saint-Martin de Sescorts $\left(2^{\mathrm{e}} \text { moitié XII }{ }^{\mathrm{e}} \mathrm{s} .\right)^{49}$. De toute évidence notre peintre a été formé dans un atelier ibérique. La simple copie de modèles n'aurait pas produit autant de similitudes au niveau de la technique picturale.

Les ressemblances avec diverses œuvres ibériques situent les peintures de Burnand entre la fin du XI ${ }^{e}$ siècle (Bible de Roda) et le début du XII ${ }^{e}$ siècle (devant d'autel de la Seu d'Urgell). La maçonnerie de l'église de Burnand a été reprise dans la seconde moitié du XI ${ }^{\mathrm{e}}$ siècle (remontage autour de la baie axiale), ce qui pourrait indiquer une rénovation intérieure motivant la production de peintures. Rappelons que le chevet de Cluny III fut mis en construction entre 1088 et 1095, et que l'arrêt du gigantesque chantier, vers 1120 , a pu favoriser la dissémination de nombreux artisans dans la région, et peut-être expliquer la présence d'un artiste ibérique à Burnand. 


\section{Gourdon, église Notre-Dame (Saône-et-Loire) et Santa Maria de Mur}

41 Les peintures murales du chœur de Notre-Dame de Gourdon sont un autre exemple de peinture hispanisante, peut-être même produite par un peintre catalan (fig. $\left.\mathbf{n}^{\circ} \mathbf{1 1}\right)$. Cet ensemble figure parmi l'un des plus importants de Saône-et-Loire, tant par les surfaces peintes (près de $200 \mathrm{~m}^{2}$ ), que par la qualité de ses peintures ${ }^{50}$.

Figure 11

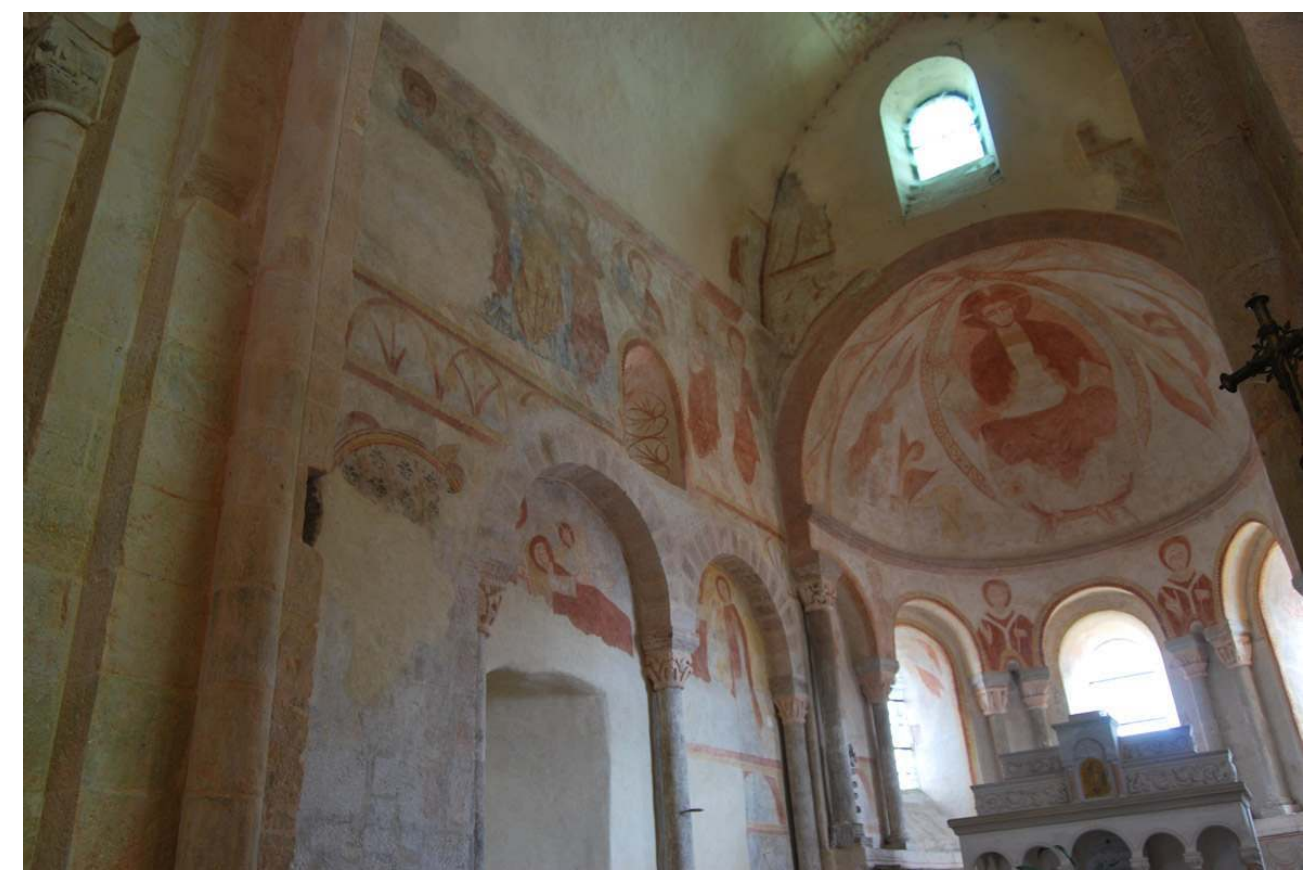

Gourdon, église Notre-Dame de l'Assomption.

PHOT. ROLLIER, J. ( ) J. ROLLIER.

C'est l'étude de la technique picturale et de certains détails qui permet d'établir des parallèles entre Gourdon et Santa Maria de Mur (Catalogne) $)^{51}$. En effet, la position des personnages, l'inclinaison des têtes et la technique picturale sont très ressemblants. De plus l'organisation schématique des vêtements, aux lignes épaisses posées sur de grands aplats uniformes, semble issue de ce même modèle catalan. Un rehaut constitué de trois traits blancs parallèles, posé sur la cuisse d'un apôtre (paroi sud) relève d'une technique similaire sur le manteau du Christ de Santa Maria de Mur. Les comparaisons restent cependant ponctuelles et ne peuvent être étendues à l'ensemble des peintures de l'abside, comme si le peintre avait eu entre les mains un carnet de modèles remplis de détails issus de plusieurs chantiers de son époque.

L'aspect anguleux des visages, la forme des yeux, soulignés par un large cerne rouge, et le menton marqué d'un cercle rouge, sont des éléments propres au maître de Gourdon, que l'on retrouve dans la peinture ibérique et dans diverses peintures du bassin de la Loire et du Berry (Méobecq ${ }^{52}$, Chalivoy-Milon ${ }^{53}$ et Montoire) ${ }^{54}$. La graphie très schématique des visages, aux yeux écarquillés, relie notre artiste à Santa Maria de Mur ${ }^{55}$ et à l'abside de la Seu d'Urgell. Le détail du cercle rouge qui apparaît sur le menton est un élément connu à Saint-Martin de Sescorts et à San Pedro de la Seu d'Urgell. Le regard entouré d'un épais 
cerne est une caractéristique qui perdure et qui se retrouve dans les peintures murales de Saint-Just à Ségovie (Castille- León) datées de la fin du XII ${ }^{\mathrm{e}}$ siècle $^{56}$.

Par ailleurs le corps nu du faune (baie sud de l'abside) de Gourdon ${ }^{57}$ peut être comparé aux nus de Maderuelo (chapelle de la Vera Cruz) et de Saint-Martin de Sescorts (peintures déposées au Musée de Vic, Catalogne) ${ }^{58}$. Nous observons les mêmes conventions graphiques dans la segmentation des parties anatomiques, des effets linéaires ou de la position des mains.

La palette picturale du maître de Gourdon est actuellement à dominantes ocre, mais il faut imaginer quelques rehauts plus riches, notamment dans la scène des Pèlerins d'Emmaüs (fig. n' ${ }^{\circ} 12$ ), où la tête du Christ se détache sur un nimbe noir qui n'est que la sous-couche d'une couleur bleue à base de lapis-lazuli ${ }^{59}$.

Figure 12

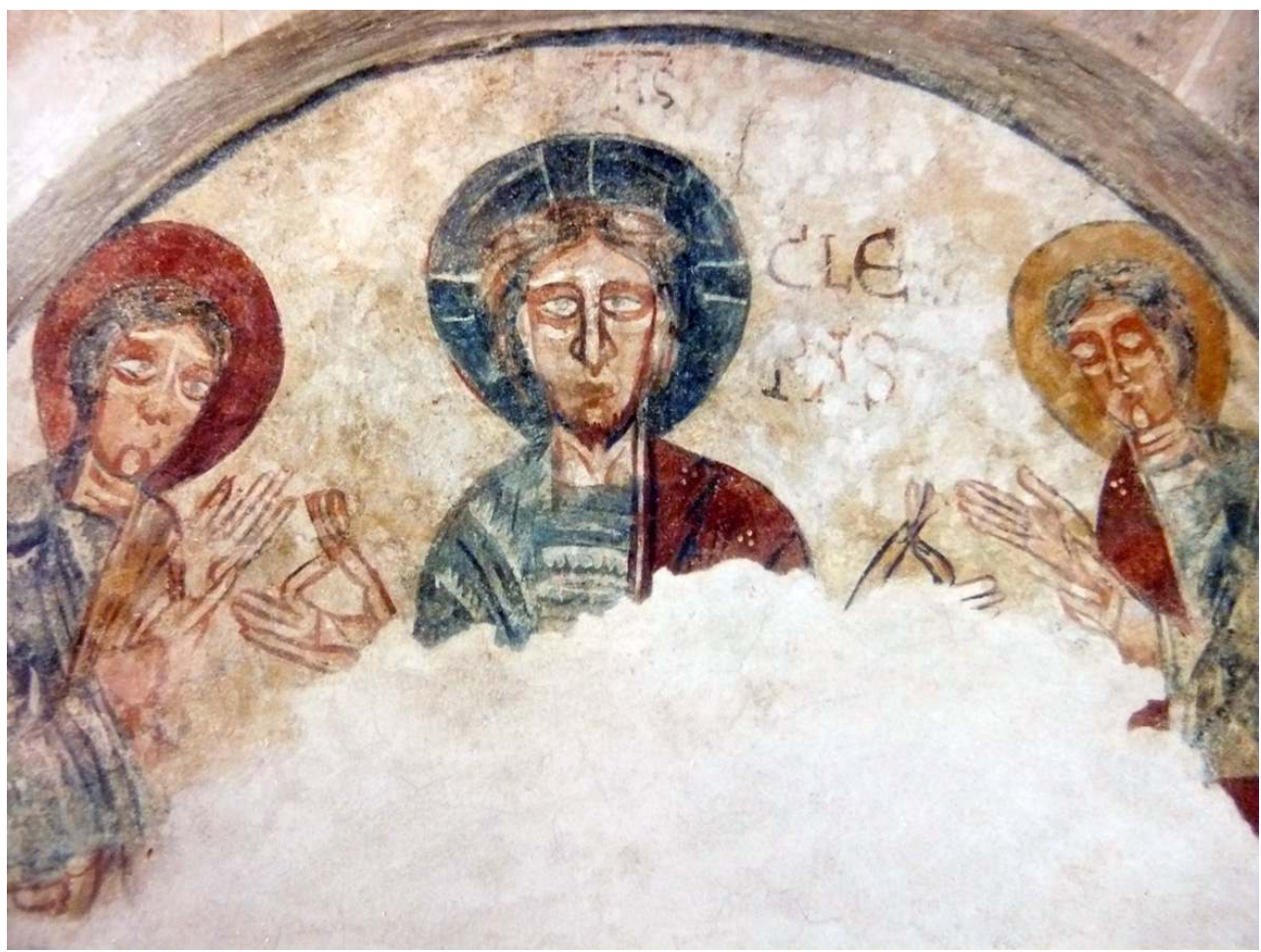

Gourdon, église Notre-Dame de l'Assomption, Pèlerins d'Emmaüs.

Phot. Rollier, J. (c) J. Rollier.

Si la formation du peintre de Gourdon semble se situer en terre ibérique, l'itinérance du peintre est également perceptible à l'église Saint-Ferréol de Curgy, près d'Autun, dont l'abside conserve un Christ en majesté, entouré du tétramorphe. Les similitudes entre les deux sites indiquent clairement qu'il s'agit du même atelier.

Quant à la datation des peintures, les études sur l'architecture et la sculpture de Gourdon ont conduit à situer la construction de l'église au début du XII ${ }^{\mathrm{e}}$ siècle $^{60}$. Les comparaisons avec les peintures ibériques ne contredisent pas cette datation, les peintures de Santa Maria de Mur étant situées entre 1112-1124 et 1151 selon les spécialistes ${ }^{61}$. L'influence de Cluny est perceptible sur l'établissement de Mur, placé sous la protection de Rome à partir de 1100, au moment où Urbain $1 l$ en confia la gestion à l'abbé Pons de Roda, qui était un rival de l'évêque Ot d'Urgell (fin Xl-début Xlle s.). Son successeur fut Ramon de 
Roda (1104-1126), ancien prieur de Saint-Sernin de Toulouse. Des relations existent d'ailleurs entre Santa Maria de Mur et la tradition picturale de la cour de Poitiers (Saint Polycarpe de Rases, Arles-sur-Tech, Serrabone, Estavat et Alet) ${ }^{62}$. Des liens étroits sont attestés entre les établissements monastiques des deux côtés des Pyrénées durant toute cette période, et le cas de Gourdon se situe probablement dans le prolongement de ce réseau.

\section{Vers un programme européen de préservation des chapelles peintes}

48 Dans certains cas, l'environnement climatique, les mauvaises conditions de conservation ou encore le tourisme de masse menacent les peintures murales. Le Landesdenkmalamt de Baden-Würtemberg (DRAC régionale) a tenté de mettre en place un projet européen de surveillance climatique et de sauvegarde de certains ensembles menacés. Les études ont montré combien les peintures ottoniennes de Saint-Georges sur l'île de Reichenau (lac de Constance) souffrent des effets climatiques du lieu et d'un tourisme effréné. Ce site-pilote a bénéficié de différentes campagnes de mesures climatiques ${ }^{63}$ qui permettent d'élargir la réflexion à d'autres sites européens, plus particulièrement ceux situés sur l'arc alpin et péri-alpin de six pays (Allemagne, Autriche, Italie, Suisse, France, Slovénie).

49 La complexité architecturale des édifices ne permet pas d'avoir une connaissance suffisamment complète des phénomènes climatiques entre les différents espaces et en relation avec les ouvertures au public. À Saint-Georges de Reichenau, de nombreuses mesures ont été faites durant les dix dernières années, à l'aide de 180 capteurs. Une méthode de réglage à distance des fenêtres a été mise en place, de manière à répondre au mieux au stress climatique. La fermeture complète de l'édifice a également été tentée, mais n'était pas idéale et présentait d'autres risques. L'aération doit être modulée en fonction de nombreux paramètres (climat, saison, nombre de visiteurs, etc.). Une cartographie 3D de tous les paramètres enregistrés devrait faire avancer nos connaissances et, à terme, permettre de proposer une gestion mieux contrôlée du site. Malheureusement, notre projet d'étude européen n'a pas été retenu.

Dans la plupart des sites peints, les spécialistes observent des altérations de différentes natures (prolifération des sels, micro-organismes, etc.). La documentation ancienne permet parfois d'évaluer le rythme des dégradations au cours des années passées, mais le plus important serait de pouvoir estimer les risques futurs encourus par les peintures selon les spécificités des conditions de conservation. La numérisation en 3D de certains édifices, comme la chapelle Saint-Martial au Palais des Papes d'Avignon, permet de centraliser la documentation ${ }^{64}$ existante sur les peintures, tout en offrant des possibilités de simulation relatives à l'utilisation du lieu. Il en résulte une base de données accessible aux conservateurs, restaurateurs et gestionnaires de sites. À terme, il est question d'offrir un outil permettant de programmer la fréquentation des chapelles en fonction des périodes climatiques les plus favorables, ou les moins risquées, pour la pérennité des œuvres.

51 L'exploitation de la documentation en 2D (photographies et documentation ancienne, constat d'état, mesures climatiques, cartographie des altérations, etc.) doit pouvoir être introduite dans le modèle 3D d'un édifice, de manière à faciliter un suivi périodique plus précis. Différentes solutions ont été mises en place en France, comme le projet NUBES, 
développé par Livio de Luca (École d'architecture de Marseille) et Jean-Marc Vallet (Centre interrégional de Conservation du Patrimoine), pour répondre à cette demande. http://www.map.archi.fr/nubes/imago/intro.htm.

Si l'utilité de ces nouveaux outils s'avère nécessaire aux différents acteurs d'un site, d'autres applications permettent une large diffusion auprès du public, de manière à le sensibiliser aux risques liés aux visites. Un exemple de navigation interactive dans les catacombes de Rome (projet ISEE, Barbara Mazzei, Pontificia commissione di archeologia sacra) conduisant à une base de données ambitieuse, est maintenant accessible (http:// VIDEMUS.it). D'autres expériences sont également en cours dans ce sens.

Il s'agit maintenant d'harmoniser les méthodes de travail entre pays européens et d'élargir ce type de travaux vers une série de peintures situées le long de l'arc alpin et péri-alpin, zone géographique plus exposée aux changements climatiques, plus particulièrement en relation avec les phénomènes de réchauffement. Différents méthodes pourraient être testées pour mieux évaluer la déformation des matériaux, en relation avec l'humidité et la température ambiante. Le développement d'un microscope 3D permettrait par exemple de mieux connaitre certains processus d'altération. Différents projets dans ce sens ont déjà vu le jour, notamment MEDISTONE (Preservation of ancient Mediterranean sites in term of their ornemental and building stone, 2003) ${ }^{65}$, SACRE (Suivi des Altérations, Caractérisation et Restauration des monuments en pierre calcaire). À terme la mutualisation des recherches et des connaissances au niveau européen devrait améliorer les connaissances et les méthodes de gestion des sites peints.

\section{NOTES}

1. - Paris, BnF, nouv. acq. lat. 2246.

2. - ROLLIER-HANSELMANN, Juliette. Les peintures murales dans les anciens territoires de Bourgogne (XI-XIIe s.) : de Berzé-la-Ville à Rome et d'Auxerre à Compostelle. Thèse de doctorat, Université de Bourgogne, décembre 2009 ; résumé dans Le Bulletin du Centre d'études médiévales d'Auxerre, ${ }^{\circ} 14$, 2010, p. 313-328 : http://cem.revues.org/.

3. - PALAZZO, Éric. « L'iconographie des fresques de Berzé-la-Ville dans le contexte de la Réforme grégorienne et de la liturgie clunisienne ». Dans Les Cahiers de Saint-Michel de Cuxa, t. 19, 1988, p. 169, 186 ; RUSSO, Daniel. «Espace peint, espace symbolique». Dans Revue Mabillon, Revue internationale d'histoire et de littérature religieuse, n.s., 11 (72),Turnhout, 2000, p. 57-87.

4. - ROLLIER-HANSELMANN, Juliette. «La tradition legis de Berzé-la-Ville: entre tradition et innovation ». Revue Hortus Artium Medievalium, Zagreb, novembre 2011.

5. - SCHILLER, Gertrud. Ikonographie der christlichen Kunst. Gutersloh, 1980, Bd 3, Abb. 580 ; CHRISTERN-BRIESENICK, B. Repertorium der christlich-antiken Sarkophage. Teil 3. Frankreich, Algerien, Tunesien. Mayence, 2003.

6. - BENOIT, F. «Sarcophages paléochrétiens d'Arles et de Marseille ». Supplément Gallia V, 1954, p. 36 ; CHRISTERN-BRIESENICK, B. Repertorium der christlich-antiken Sarkophage. Teil 3. Frankreich, Algerien, Tunesien. Mayence, 2003, p. 39-40. 
7. - BERTELLI, Carlo, BRAMBILLON BARCILON, Pinin. Il ciborio della basilica di Sant'Ambrogio in Milano. Milan, 1981 ; CAILLET, Jean-Pierre, GABORIT-CHOPIN, Danielle, PALAZZO, Éric. L'Europe de l'an mil. Orléans : Zodiaque, 2001, p. 234.

8. - COE, Almestad. The Sculpture of Saint-Sauveur de Nevers. Thèse, University of California, Berkeley, 1987.

9. - FRANZE, Barbara. La pierre et l'image. L'église de Saint-Chef-en-Dauphiné. Paris : Picard, 2011.

10. - MAIER, Jean-Louis. Le baptistère de Naples et ses mosaïques. Étude historique et iconographique. Fribourg : Ed. universitaires, 1964.

11. - VIVIER, Marion. Les fresques romanes de Saint-Chef. Grenoble, 2000 ; FRANZE, Barbara. La pierre et l'image. L'église de Saint-Chef-en-Dauphiné. Paris : Picard, 2011.

12. - DAVY, Christian, JUHEL, Vincent, PAOLETTI, Gilbert. Les peintures murales romanes de la vallée du Loir. Vendôme : Cherche Lune, 1997, p. 134-147, p. 141.

13. - ROLLIER-HANSELMANN, Juliette. «Découverte d'un Christ caché sous les repeints ». Bulletin monumental, t. 163-3, 2005, p. 243-249.

14. - ROLLIER-HANSELMANN, Juliette. "Peintures et couleurs dans la mouvance clunisienne ". Peintures murales médiévales XIIe-XVIe s. Regards comparés (D. Russo, dir). Dijon : Ed. universitaires, 2005, p. 113-127.

15. - SENDLER, Egon. L'icône - Image de l'invisible. Paris : Istina Syracuse, 1981, p. 151.

16. - KIRSCHBAUM, Engelbert. Lexikon der christlichen Ikonographie. Freiburg-im-Breisgau : éd. H. Kirschbaum, éd. Herder, 1968-76, Band 2, p. 10.

17. - JAMES, Liz. Light and Colour. Oxford : Clavendon Press, 1996.

18. - FAVREAU, R. (dir.). Saint-Savin, l'abbaye et ses peintures murales. Poitiers, 1999, p. 126, photos p. 123-124.

19. - SUCKALE, Robert. Das mittelalterliche Bild als Zeitzeuge. Berlin: Sechs Studien, 2002 et ROMANO, Serena. Riforma et tradizione, 1050-1198. La pittura medievala a Roma, Corpus, vol. IV, Rome : Jaca Books, 2006.

20. - RICCIONI, Stefano. Il mosaico absidale di S. Clemente a Roma-Exemplum della chiesa riformata. Spolète : Fondazione Centro italiano di studi sall'alto medievo, 2006 ; ROMANO, Serena. Riforma et tradizione, 1050-1198. La pittura medievala a Roma, Corpus, vol. lV. Rome : Jaca Books, 2006.

21. - ROLLIER-HANSELMANN, Juliette. «Découverte d'un Christ caché sous les repeints ». Bulletin monumental, t. 163-3, 2005, p. 243-249.

22. - Voir article sur le sujet: ROLLIER-HANSELMANN, Juliette. «Géométrie et modules de construction à l'époque romane: de Constantinople à Berzé-la-Ville». Actes du colloque Arch-ITech, Cluny : Ausonius, 2011.

23. - DAVY, Christian. La peinture murale romane dans les pays de Loire. Laval: Société d'archéologie et d'histoire de la Mayenne, suppl. 10, 1999.

24. - HISCOCK, Nigel. The Symbol at your Door, Number and Geometry in Religious Architecture of the Greek and Latin Middle Ages. Adelshot, 2007, p. 65 ; HOFFMANN, Volker. Der geometrische Entwurf der Hagia Sophia in Istanbul. Bilder einer Ausstellung. Berne : Peter Lang, 2005 ; LANC, Elga. Romanische Wandmalereien in der Burgkapelle Ottenstein. Vienne ; 1000 Jahre Babenberger in Österreich. Katalog der Niederösterreichischen Jubiläumsausstellung im Stift Lilienfeld vom 15. Mai bis 31. Oktober 1976. Veranstaltet vom Bundesland Niederösterreich. Éd. Erich Zöllner, Karl Gutkas, Gottfried Stangler, Gerhard Winkler. Katalog des Niederösterreichischen Landesmuseums. N.F. 66. - Wien, 1976, XXIII, 774. $8^{\circ}$. Objekt-Nr.: 922a, S. 503. http://wwwg.uni-klu.ac.at/kultdoku/kataloge/11/ html/1011.htm.

25. - Dijon, Bib. Mun, ms. 12-15 - CAHN, Walther. Romanesque Manuscript Painting in France. Londres : Brepols,1996, ill. 133-134, cat. 58.

26. - LABBÉ, Alain. «Contribution à l'interprétation de la fresque du Christ à cheval de la crypte de la cathédrale Saint-Étienne d'Auxerre: notes sur l'iconographie impériale ottonienne, le concept théologique de royauté du Christ et la majesté équestre de Charlemagne dans la chanson 
d'Aspremont ». Bulletin de la Société Française d'Archéologie de l'Yonne, 3, 1986, p. 73-79; id. « Nouvelle contribution à l'interprétation de la fresque du Christ à cheval de la crypte de la cathédrale Saint-Étienne d'Auxerre: deux résurgences carolingiennes du thème antique de l'Adventus Augusti, Hugues de Chalon et Otton III ». Bulletin de la Société Française d'Archéologie de l'Yonne, 4, 1987, p. 57-68.

27. - DON DENNY. «A Romanesque Fresco in Auxerre Cathedral». Gesta, 25, 1986, p. 197-202 ; FRANZE, Barbara. « Les peintures romanes de la cathédrale d'Auxerre. Une relecture ». Bulletin du Centre d'études médiévales d'Auxerre, $\mathrm{n}^{\circ} 14,2010$.

28. - FRANZE, Barbara. «Des peintures de Nevers aux œuvres de la réforme : les témoins d'une tradition iconographique ». Bulletin du Centre d'études médiévales d'Auxerre, n¹2, 2008.

29. - Pour un état de la question, voir SUBES, Marie-Pasquine. « Note sur l'étude des peintures murales médiévales à l'inventaire général ». Monumental n²0, Peintures murales (2). Paris, 1998, p. 88-91.

30. - Les peintures murales de Poitou-Charentes. BROCHARD, Bernard (dir.), RIOU, Yves-Jean. « SaintSavin : Centre International d'Art Mural ", 1993 ; Saint-Savin, l'abbaye et ses peintures murales. FAVREAU, Robert (dir.) rééd. FAVREAU, Robert, JEANNEAU, François, RIOU, Yves-Jean, et al.. Poitiers: Connaissance et promotion du patrimoine de Poitou-Charentes, 1999. http:// inventaire.poitoucharentes.fr/ressources/publications/pdf/

publication_itineraires_peinturesmurales_poitou.pdf.

31. - COURTILLE, Anne. Histoire de la peinture murale dans l'Auvergne du Moyen Age. 1983.

32. - DAVY, Christian. La peinture murale romane dans les Pays de la Loire. 1999.

33. - KUPFER, Marcia. Romanesque Wall painting in Central France. New Haven : Yale Publications in Art History, Londres, 1993.

34. - Thèse en cours d'Anne Leturque à l'Université de Montpellier 3, sous la direction de Géraldine Mallet.

35. - DUBY, Georges. La société aux XIe-XIIe s. dans la région mâconnaise. Paris : EHESS, 1971, p. 355.

36. - Saint-Isidore de Duenas en 1073, Saint-Zoilo de Carrion de los Condes en 1076, Santa Maria de Najera en 1079 et Santa Colomba de Burgos en 1081. HENRIET, Patrick. « Un bouleversement culturel. Rôle et sens de la présence cléricale française dans la péninsule ibérique $\left(\mathrm{XI}^{\mathrm{e}}-\mathrm{XII}{ }^{\mathrm{e}}\right.$ siècles) ». Société d'histoire religieuse de la France, vol. 90, 1, 2004, p. 65-80.

37. - CALLAGHAN, O. "The integration of Christian Spain into Europe: the role of Alfonso VI of Leòn-Castille». Dans REILLY, F. (dir.) Santiago, St Denis and St Peter. The Reception of the Roman Liturgy in Leon-Castille in 1080, New York, 1985, p. 101-120 ; LÉONET, Guy, « Cluny, une coquille vide? », 1998, op. cit., p. 146 ; BOTO VARELA, Gerardo, « Monasterios Catalanes en el siglo XI. Los espacios ecclesiasticos de Oliba», Monasteria et Territoria, Elites, edilicia y territorio nel Mediterraneo medieval (siglos V-XI). Madrid (2006), 2007.

38. - CAHN, Walther. Romanesque Manuscripts, op.cit., 1996, vol. II, p. 69-70 ; SCHAPIRO, Meyer. The Parma Ildefonsus, op.cit., 1964 ; ANIEL, Jean-Pierre. " Le scriptorium de Cluny aux Xe et XIe s. ». Le gouvernement d'Hugues de Semur à Cluny, Actes du colloque scientifique. Cluny, 1988, p. 265-282, notamment p. 275.

39. - COCCHETTI-PRATESI, Lorenza. Il Parma Ildefonsus, Cluny e la pittura cataluna. Dans «Arte Lambarda », n.s. LII, 1979, p. 21-30. 1979 ; ZANICHELLI, Giuseppa. « Strutture narrative a Cluny : Il Parma Ildefonsus ». Medioevo : immagine e racconto, 2000.

40. - L'image se trouve au-dessus de la porte d'entrée, au revers de façade. La peinture, restaurée par Anne Féton, est en bon état de conservation et n'a pas été repeinte, ni retouchée. L'inscription fragmentaire ....OS(ET) MORTVOS IND....US, autour du médaillon, semble faire allusion à des rites funéraires.

41. - PAGES I PARETAS, Montserrat. Sobre pintura romanica catalana, Abbaye de Montserrat, 2005. p. $147-158$. 
42. - VIREY, Jean. Les églises romanes, 1934, p.112-113 et observations de Gilles Rollier, archéologue.

43. - Elles ont été restaurées entre 1985 et 1988, sous la direction d'Anne Féton (Chantier de l'association "Intérieurs Historiques de Bourgogne ») en quatre campagnes avec des stagiaires bénévoles.

44. - FETON, Anne. «Les peintures murales de Burnand». Images de Saône-et-Loire, $\mathrm{n}^{\circ}$ 72, 1987, p. 14 .

45. - WILLIAMS, John. The Illustrated Beatus. A Corpus of the Illustrations of the Commentry on the Apocalypse, vol. IV, 11-12 ${ }^{\text {th }}$ cent. Londres-Turnhout, 2002 ; DE PALOL, Père, HIRMER, Max. L'art en Espagne. Paris, 1967, catalogue $\mathrm{n}^{\circ} 83$.

46. - DE PALOL, Pere, HIRMER, Max. L'art en Espagne, op.cit., 1967, p. 160.

47. - ROLLIER-HANSELMANN, Juliette. «Els murals hispans de Borgonya: els exemples de Burnand i Gourdon » (« Peintures murales hispanisantes de Bourgogne : les exemples de Burnand et Gourdon ». Rivista cultural dell' Alt Urgell, URTX. Tarrega, 2011, p. 180-197.

48. - CAHN, Walther. La Bible romane. Fribourg, 1982, p. 72 ; Peter Klein (1972) situe le manuscrit entre le second tiers et troisième quart $\mathrm{du} \mathrm{Xl}^{\mathrm{e}} \mathrm{s}$; YARZA LUACES, Joaquin. "Peregrinacion a Santiago y la pintura y miniatura romanicas ». Compostellarum, 30, 1985, p. 369-393.

49. - SUREDA, Joan. La pintura romanica en Cataluna. Madrid, 1995.

50. - KAGAN, Judith. "Gourdon, église de l'Assomption de la Sainte Vierge ", D'ocre et d'azur, Peintures murales en Bourgogne. Dijon: Musée archéologique, 1990; ROLLIER-HANSELMANN, Juliette. "Iconographie, style et technique des peintures murales de l'église Notre-Dame de Gourdon ». Dans REVEYRON, N. (dir.). Le renouveau des études romanes, $2{ }^{\text {ème }}$ colloque scientifique international de Paray-le-Monial, 2000, p. 267-282 ; ROLLIER-HANSELMANN, Juliette. « Gourdon, Église Notre-Dame ». Congrès archéologique de France, Paris, 2008.

51. - «Peintures déposées durant l'été 1919 à l'insu des autorités espagnoles ». MEISLER, S. Smithsonian Magazine, avril 1998 ; PAGES I PARETAS, Montserrat. Sobre pintura romanica, op.cit., 2005 , p. 129.

52. - VERGNOLLE, Éliane. «Peinture et architecture : l'ancienne église abbatiale de Méobecq ». Cahiers de l'inventaire, 1988.

53. - KUPFER, Marcia. op.cit., note 32.

54. - DAVY, Christian, JUHEL, Vincent, PAOLETTI, Gilbert. Les peintures murales de la vallée du Loir. Vendôme, 1997 ; voir d'autres exemples dans DAVY, Christian. La peinture murale romane dans les Pays de la Loire. Laval, 1999.

55. - PAGES I PARETAS, Montserrat. Sobre pintura romanica catalana. Abadia di Montserrat, 2005, p. 126. Les peintures sont datées entre 1069 et 1100.

56. - GRAU LOBO, Luis. Pintura romanica en Castilla y León. León, 2001, p. 1254 ; FERNANDEZ SOMOZA, Gloria. « Martirio e inventio de los Santos Ninos de Compluto : las pinturas murales de San Justo de Segovia ». Boletin de $l$ museo e instituto Camon Aznar, $\mathrm{n}^{\circ}$ 80, 2000, p. 123-140; MARTINEZ BARGUENO, Manuel. Las pinturas murales de San Justo de Segovia. Août 2009. http:// manuelblasmartinezmapes.blogspot.com/2009/08/las-pinturas-romanicas-de-san-justo-en.html 57. - Adam sur l'arc triomphal, Faunus de la fenêtre sud.

58. - DEMUS, Otto, HIRMER, Max. La peinture murale romane. Paris, 1970, fig. 173, p. 156. Les peintures de Maderuelo sont datées du début du XII ${ }^{\mathrm{e}}$ siècle.

59. - ROLLIER-HANSELMANN, Juliette. «D'Auxerre à Cluny: technique de la peinture murale entre le Vllle et le Xlle siècle en Bourgogne ». Cahiers de civilisation médiévale, 40. Poitiers, 1997, p. $57-90$.

60. - REICHE, Jens. «Le décor sculpté de Gourdon et de Mont Saint-Vincent : un atelier charolais du début du XIIe s. ". Dans REVEYRON, Nicolas (dir.). Le renouveau des études romanes, 2 ème colloque scientifique international. Paray-le-Monial, 2000, p. 239-255.

61. - PAGES I PARETAS, Montserrat. Sobre pintura, op cit., 2005, p. 127-128. 
62. - FERNANDEZ SOMOZA, Gloria. Pintura romanica en el Poitou, Aragon y Cataluna. La itinerancia de un estilo. Nausicaa, 2005 ; PAGES I PARETAS, Montserrat. «Les pintures de Santa Maria de Mur, seu d'una canonica fundad pels comtes de Pallars Jussa ", éd. R. Alcoy i P. Besaran, El romanic i el Gotic desplaçats. Estudis sobre l'exportacio I migracions de l'art catala medieval, Université de Barcelone, 2007, p. 19-54. Le style du peintre de Mur diffère sensiblement des autres productions catalanes de la même époque (Pedret, Tahull, Osormort), mais des ressemblances existent avec celle de la Seu d'Urgell et S. Miguel de Moror.

63. - JAKOBS, Dörthe, REICHWALD, Helmut F. « Untersuchungsergebnisse und Massnahmen der jüngsten Restaurierung von St Georg, Reichenau-Oberzell ». Zeitschrift für Kunsttechnologie und Konservierung. Heft, 2, 4, Worms, 1990.

64. - VALLET, Jean-Marc, DE LUCA, Livio, GUILLON, Odile, PIERROT-DESEILLIGNY, Marc, BAUDRY, Olivier, TRABELSI, Nassim. "An interactive 3-dimensional database applied to the conservation of a painted chapel ». Proceedings of 7th International Conference on Science and Technology In Archaeology and Conservation. Workshop on Documentation and Conservation of Stone deterioration in Heritage Places. Petra (Jordan), December 7 to 12, 2010.

65. - Voir le site : http://www.lrmh.fr/lrmh/telechargement/cs/pierre_2009.pdf.

\section{RÉSUMÉS}

Le corpus des peintures murales romanes des anciens territoires de Bourgogne comprend vingtcinq sites, situés dans une vaste aire géographique allant d'Auxerre à Bâle pour la limite nord, et de Nevers à la Provence pour la partie méridionale. Dans un premier temps, nous évoquerons l'état des recherches concernant la Chapelle-des-Moines de Berzé-la-Ville (iconographie, style, technique). Dans un second temps, nous présenterons rapidement quelques peintures influencées par l'ouest de la France, tandis que la troisième partie traitera de peintures de style hispanisant. Enfin nous terminerons par la présentation de l'état de conservation de certains sites.

The corpus of romanesque wall paintings in the ancient territories of Burgundy is actually of twenty-five sites, situated in a huge area going from Auxerre to Basel for the northern limit, and from Nevers to Provence for the southern part. In a first time, we present the actual state of research for the Chapelle-des-Moines at Berzé-la-Ville (iconography, style, technique). In a second time, the interest is put on paintings influenced by west France and we will finish with paintings showing Hispanic style. The conservation of some sites will end our purpose.

\section{INDEX}

Mots-clés : peinture murale romane, Bourgogne, Cluny, Espagne

Keywords : romanesque wall painting, Burgundy, Cluny, Spain 
AUTEUR

JULIETTE ROLLIER-HANSELMANN

Docteur en histoire de l'art et restauratrice de peintures, Responsable restitution architecturale, Équipe Gunzo, Arts et Métiers ParisTech, Cluny juliette.rollier@ensam.eu 